\title{
The effect of low dose pethidine and diazepam for sedation in Cesarean Section under Sub-Arachnoid Block on mothers' satisfaction
}

\author{
DILIP KUMAR SAHA ${ }^{1}$, MAZIBAR RAHMAN $^{1}$, PARESH CHANDRA SARKAR ${ }^{2}$, DEBASISH BANIK ${ }^{3}$, \\ QUAMRUL HUDAAK ${ }^{3}$, FIROZA BEGUM ${ }^{4}$
}

\begin{abstract}
Objective: Cesarean Section (CS) is usually performed when a vaginal delivery would put the baby's or mother's life at risk. Regional anesthesia has the advantage over general anesthesia by allowing mother to remain awake during operation. However, some women prefer general anesthesia as they want to be asleep during the operation. . Aim of our study was to see the effect of mild sedation on mothers' satisfaction during CS.

Materials and methods: A prospective cross sectional study was undertaken in the department of Anesthesiology, Institute of Child and Mother Health from January 2009 to December 2009. Sixty patients of singletone pregnancy with ASA(American Society of Anesthesiology)-I were randomized into two groups I\&II of which one group received sedation with low dose pethidine and diazepam and other group did not get any sedation.
\end{abstract}

Results: Data were analyzed between the groups and within the groups using students' $t$ ' test and Chi-Square test. A p-value of $<0.05$ was considered as significant. Patient's parameters like heart rate and blood pressure were not statistically significant between groups, but $\mathrm{SpO}_{2}$ fall was statistically significant. Mother's satisfaction is significantly high (76\%) in sedation group than non sedation group.

Conclusion: By sedation with low dose pethidine and diazepam, mothers' satisfaction was better achieved without affecting the hemo-dynamic stability.

Keywords: sedation, cesarean section, sub-arachnoid block, mothers' satisfaction.

Introduction:

A Cesarean Section (CS) is usually performed when a vaginal delivery would put the baby or mother's life at risk. Regional anesthesia has the advantage over general anesthesia by allowing mother to remain awake during delivery. Besides this, anesthesia related maternal mortality is also decreased when CS is done under regional anesthesia ${ }^{1,2}$. In UK, The fall in maternal mortality was 12.8 to 1.7 per one million live births and in USA, it was 4.3 to 1.9 per one million live births between the late 1970s and the late 1980s. This is believed to be partly due to the increasing use of regional anesthesia for cesarean delivery ${ }^{3}$.

Therefore, SAB (Sub Arachnoid Block) for CS is becoming popular to the anesthesiologists considering the benefits to the mother and her fetus.

Pethidine is a synthetic opioid with sedative effects. It can produce prompt but short-lasting analgesia. It is easily available and we are familiar with its use in our everyday practice. In low dose it has an antishivering effect also. There are antidotes (naloxone) available in case of overdoses.

Diazepam is a benzodiazepine, cheap and easily available. Because of its lipid solubility it can easily cross the blood-brain barrier and can produce prompt sedation. Flumazenil is available to reverse it's over sedation.

The purpose of sedation during spinal anesthesia is to alleviate anxiety and prevention of any body movement. It also provides an appropriate degree of memory loss or decreased awareness after the procedure $^{5}$. There was no consensus of sedation protocol for CS under spinal anesthesia. In Hong Kong, the Co- coordinating committees in pediatrics, radiology and anesthesiology (COC) has formulated a set of guidelines for sedating children for diagnostic

1. Assistant Professor, Department of anesthesia, Institute of Child and Mother Health,

2. Assistant Professor, Department of Anesthesia, Sir Salimullah Medical College, Mitford Hospital,

3. Associate Professor, Department of Anesthesia, Analgesia and Intensive Care Medicine, BSMMU,

4. Professor, Department of Obstetrics \& Gynaecology, BSMMU. 
and therapeutic procedures in year $2000^{4}$.In the COC guidelines, one recommendation is to give pethidine $0.5 \mathrm{mg} / \mathrm{kg}$ intravenously, titrated with midazolam at $0.025-0.2 \mathrm{mg} / \mathrm{kg}^{4}$.

Sedation is drug induced state ranging from responding verbal commands to loss of consciousness. ${ }^{8}$ Moderate sedation/ analgesia were the most common target level of sedation used in non invasive procedure. Clinical Manual states that optimal moderate sedation level is achieved when the patients- maintains consciousness, independently maintains airway; retain protective reflexes and responds to verbal and physical commands ${ }^{5}$.

Where there is combination of sedative and analgesic treatment, there would be predilection to cause respiratory depression and airway obstruction. The Practice Guidelines for Sedation and Analgesia by the Non- anesthesiologist formulated by the ASA emphasized the need to appropriately reduce the dose of each component as well as need to continuously monitor respiratory and circulatory function ${ }^{7}$.

Several sedation scales and scoring systems have been developed to describe the level of sedation. Modified Observer's Assessment of Alertness/ Sedation Scale and Ramsey Sedation Scale Score are usually used in clinical research. ${ }^{8}$

In spite of extensive literature search, no universally adopted sedation protocol during cesarean section under SAB was found. We adopted the combination of intravenous pethidine and diazepam as the sedation protocol. The aim of this study is to explore/ assess the satisfaction of mother undergoing CS operation under spinal anesthesia.

\section{Methods:}

The institutional ethical committee approval and written informed consent from sixty (60) patients were obtained for this descriptive randomized controlled clinical trial (DCT). The patients had the normal history of singleton pregnancy and an ASA physical status I. Pre-anesthetic assessments were done on the day before surgery. The patients with suspected or who had bleeding disturbances, gross abnormality in vertebral column, infection in the back, presence of liver and kidney diseases, patients taking anticoagulant and patient with pregnancy induced hypertension $(\mathrm{PIH})$ or preeclampsia were excluded from the study. The patients selected for the study was divided into two groups; Inj. prochlorperazine $5 \mathrm{mg}$ was given to both groups of study populations. GroupI(with medication): 30 patients, Group -II (without any drugs): 30 patients. The patients were informed about the study and the procedure and selected through lottery methods - in cases (Group-I) and controls (Group-II).

Once the patient was brought to the theatre, she was allowed to lie down into the left lateral position. The baseline blood pressure, heart rate, $\mathrm{Spo}_{2}$ were measured and recorded. An intravenous channel was established with wide bore I/V canula and then the patient was pre-loaded with Hartmann's solution at a rate of $10 \mathrm{ml} / \mathrm{kg}$.body weight in 20- 30 minutes. According to the number of the token which she obtained on the previous day, anesthetic technique was employed.

A quinke babcock 27-G spinal needle was used at the L3-4 interspace in the sitting position. After spinal anesthetic technique, the patient was turned to supine position and a pillow was placed under the head. The operating table immediately tilted 15 degree to the left. The patients of group- 1 received intravenous diluted 20 mg pethidine titrated with $2.5 \mathrm{mg}$ diazepam just after the delivery of fetus of group II received no sedation

Mothers' satisfaction was measured using the following scale (maternal satisfaction score, 2 points for each parameter $)^{9}$ by the investigator during interview of the mother at the end of operation.

Level of mothers' satisfaction:

\begin{tabular}{llll}
\hline Parameter & Score-2 & score-1 & score-0 \\
\hline Nausea/vomiting & No nausea & Nausea & Vomiting \\
Chest pain & No pain & Heaviness & Pain \\
Restlessness & Calm \& quiet & Apprehended & Restless \\
Limb discomfort & No & Mild & Severe \\
Shivering & No & Mild & Mild \\
\hline
\end{tabular}

Highly satisfied (8-10); fairly satisfied (5-7); not satisfied $(<5)$

\section{Results:}

For statistical analysis, the quantitative unpaired student 't' test and Chi Square test for qualitative data were used with SPSS Version 9.0. $P<0.05$ was considered significant.

During operation, there was mild fall of Blood Pressure (BP) in both groups. There was no significant difference between two groups regarding fall in BP that was needed to be treated with vasopressor. Maternal satisfaction is significantly high $(76 \%)$ in sedation group than non sedation group. After interview of mothers at the end of operation, maximum (90\%) mothers recommended for sedation as a satisfactory technique during cesarean section operation under spinal anesthesia whereas (70\%) of mothers without sedation (group II) express their dis-satisfaction about the procedure. 
Table-I

Comparison of intra-operative events

\begin{tabular}{lcccc}
\hline Variables & Group-I(n=30) & Group-II $(\mathrm{n}=30)$ & Chi-square & P value \\
\hline Hypotension (needed to be & $10(33 \%)$ & $04(12.5 \%)$ & 1.06 & 0.30 \\
treated with vasopresso/fluid). & & & & \\
Shivering & $02(6.66 \%)$ & $07(23.33 \%)$ & & 0.15 \\
Epigastric pain & $09(30 \%)$ & $15(50 \%)$ & 2.50 & 0.11 \\
$\mathrm{SpO}_{2}$ fall to 90\% & $12(40 \%)$ & $01(3.33 \%)$ & 11.68 & 0.0006 \\
\hline
\end{tabular}

$\mathrm{P}>0.05-$ not significant.

Table-II

Level of patient's satisfaction

\begin{tabular}{lcccc}
\hline Patient satisfaction & Group-I $(\mathrm{n}=30)$ & Group-II $(\mathrm{n}=30)$ & Chi-square & $\mathrm{P}$ \\
\hline Highly satisfied & $23(76.66 \%)$ & $03(10 \%)$ & 27.15 & $<.001$ \\
Fairly satisfied & $07(23.33 \%)$ & $12(40 \%)$ & 1.93 & 0.17 \\
Not satisfied & $00(0 \%)$ & $15(50 \%)$ & 20.00 & $<.001$ \\
\hline
\end{tabular}

$P<0.05$ - significant; $P<0.01$ - highly significant.

Table-III

Postoperative interview of patients

\begin{tabular}{lcccc}
\hline Variables & Group-I(n=30) & Group-II $(\mathrm{n}=30)$ & Chi-square & $\mathrm{P}$ \\
\hline Pain/Discomfort & $4(13.33 \%)$ & $21(70 \%)$ & 19.82 & $<0.001$ \\
Choice of technique & $26(86.66 \%)$ & $14(46.66 \%)$ & 10.80 & $<0.001$ \\
Recommendation & $27(90 \%)$ & $15(50 \%)$ & 11.43 & $<0.001$ \\
\hline
\end{tabular}

$\mathrm{P}<0.01$ - is highly significant.

\section{Discussion:}

The sub-arachnoid block is the single widely used and accepted technique for cesarean section operation. But not without unwanted experience like nausea, vomiting, chest pain, restlessnss, lower limb discomfort, shievering and so on. We have considered these parameters to level mother's comfort and overall mothers' satisfaction. The present and previous experiences of mothers were also compared .Recommendation of the mothers about anesthetic technique for CS has also been taken into account.

There was no consensus of sedation protocol for patients of sub-arachnoid block undergoing CS operation. We have shown that the combination of low dose intravenous pethidine and diazepam is an effective and safe sedation protocol for this purpose.

The safety profile of our sedation protocol is comparable to Riavis's study which quoted a $10 \%$ of cases suffered from transient oxygen desaturation while our result showed that $13,3 \%$ cases has mild oxygen desaturation. ${ }^{7}$ In all cases having oxygen desaturation, they had good response to oxygen supplement. Transient fall in Spo2 up to 90\% has no significant effect on patient's tissue oxygenation as well as fetal well-being's. ${ }^{6}$

In our study, we use intravenous pethidine $0.4-0.5 \mathrm{mg} /$ $\mathrm{kg}(20 \mathrm{mg})$ titrate with diazepam 0.025-0.1 mg/kg (2$2.5 \mathrm{mg}$ ) with close monitoring of patients by pulseoximetry. Standby oxygen supplementation was kept ready for safety precaution in sedation. These findings were consistent with the recommendations in American Society of Anaesthesiologists ${ }^{8}$.

\section{Conclusion:}

Peri-operative wellbeing of the patients depends on several factors- of which the most important one is mental status and attitude of patients towards the whole procedure. With mild to moderate degree of sedation, the maternal satisfaction is better achieved without affecting the hemo-dynamic stability. 


\section{References:}

1. Hawkins JL, Koonin LM, Palmer SK, Gibbs CP. Anesthesia-related deaths during obstetric delivery in United States 1979-1990, Anesthesiology 1997; 86(2):277-84.

2. Hibbard BM, Anderson MM, Drife JO, Tighe JR, Gordon G, Willatts S, et al. Deaths associate with anesthesia. In: Rubery E, Bourdillon $\mathrm{P}$ editor(s). Reports on confidential enquiries into maternal deaths in the united Kingdom 19911993. Norwich: HMSO 1996: 87-102.

3. Ezri T, Szmuck P, Evron S, Geva D, Hagay Z, Katz J. Difficult airway in obstetric anesthesia: a review. Obstetric and Gynaecological Survey 2001;56(10):631-41.

4. Guidelines for sedating children for diagnostic and therapeutic procedures. The Coordinating
Committees in Paediatrics, Radiology and Anaesthesiology, Hong Kong, 2000.

5. http:// www.sedationfacts.org/sedationadministration/sedation-levels.

6. Riavis M, Laux-End R, Carvajal-Busslinger MI, Tschappeler H, Bianchetti MG. Sedation with intravenous benzodiazepine and ketamine for renal biopsies. PediatrNephrol 1998;12:147-8.

7. American Society of Anesthesiologists Task Force on sedation and Analgesia by nonAnesthesiologists.Practice guidelines for sedation and analgesia by non-anesthesiologists. Anesthesiology 2002;96:1004-17.

8. American Society of Anesthesiologsts. ASA Standards, Guidelines and Statements, October 2007. http//www2.asahq.org/publications/p-106asa-standards-guidelines-and-statements-aspx. 